\title{
Docencia e investigación en Medicina Legal: Situación actual y desafíos para las Facultades de Medicina de Chile
}

\author{
Leonardo G onzález $\mathrm{W}^{1,2,3}$, José Antonio Inzunza Na, \\ Luis Bustos $M^{2, b}$, Carlos Vallejos $V^{2}$, René G utiérrez $L^{1,4}$. \\ Training and research in Forensic \\ Medicine: Present situation and \\ future challenges
}

Background: Lawyers need some medical knowledge and physicians must know about forensics. Aim: To explore training and research programs in forensic medicine in Chilean universities. Material and methods: Deans of all Medicine Faculties in Chile were contacted by e-mail and invited to answer a questionnaire containing 21 questions. A survey of Chilean publications on forensic medicine was performed in Medline, Lilacs and SciELO databases. Results: Fourteen deans answered the questionnaire. In all the responding faculties, forensic medicine is an obligatory course, generally during the fifth year and mostly combining theory with practice. In seven faculties, forensic medicine concepts are included in other courses. Forensics is taught in only two of 10 dental schools, two of 17 nursing schools, one of nine midwives schools and one of nine medical technology schools. It is not taught in phonoaudiology, kinesiology and nutrition schools. There are 74 physicians that teach the specialty but only 10 are certified by the National Board of Medical Specialty Certification (CONACEM). Treatment of most topics on forensics is insufficient. Thanatology is the strongest topic and forensic dentistry is the weakest. There are 52 publications in the area, mostly on "medical law". Conclusions: Forensic medicine is taught in medical schools mostly as thanatology. The knowledge of forensics among medical students is limited and must be improved (Rev Méd Chile 2005; 133: 805-12).

(Key Words: Education, medical; Forensic medicine; Thanatology)

Recibido el 8 julio, 2004. Aceptado el 10 mayo, 2005.

${ }^{1}$ Instituto de Investigación Médico Legal (INIMEL), Temuco, Chile. ${ }^{2}$ CIGES (Capacitación, Investigación y Gestión para la Salud Basada en Evidencia), Temuco, Chile. ${ }^{3}$ Departamentos de Especialidades Médicas y ${ }^{4}$ de Cirugía y Traumatología, Facultad de Medicina, Universidad de La Frontera, Temuco, Chile.

anterno Carrera de Medicina, Universidad de La Frontera, Temuco, Chile.

bioestadístico.

Correspondencia a: Dr. Leonardo González W. Instituto de Medicina Forense, Johann Wolfgang Goethe Universität. Kennedyalle 104, D-60596, Frankfurt, Alemania. Fonos: 4969-63017553 y 49-69-67734888, Fax: 49-69-63015882.

E-mail: lagista@gmail.com 
$\mathrm{M}$ edicina y derecho parecen disciplinas un tanto divergentes, pero existe un puente: la medicina legal (ML). Juristas y legisladores necesitan el conocimiento médico y los profesionales de la salud necesitan el conocimiento legal ${ }^{1}$. Esta última necesidad se expresa en 2 niveles:

a) Deontológico: todas las profesiones de la salud requieren conocimientos sobre las leyes y elementos éticos que rigen la actividad profesional.

b) Pericial y forense: médicos, odontólogos y otras profesiones del área, deben poseer una sólida formación médico legal, que les permita desempeñarse adecuadamente en materias médico-legales o como peritos.

El escenario actual plantea cambios que ponen de manifiesto esta necesidad de conocimiento. Por una parte, la judicialización del ejercicio médico y el aumento exponencial de reclamaciones por malpraxis, demandan una profundización en la formación deontológica ${ }^{2-9}$. Por otro lado, la progresiva implementación de la Reforma Procesal Penal, exige fortalecer el nivel pericial y forense, máxime cuando varias disposiciones legales ${ }^{10-12}$ establecen que la actividad pericial no es privativa de los especialistas que laboran en el Servicio Médico Legal.

Producto de estos cambios en el entorno socio cultural, las experticias en ML constituyen hoy más que antes una necesidad de país, que como tal, debe ser asumida por las entidades formadoras a nivel de pre y postgrado ${ }^{13-17}$. Este desafío debe incluir también a la investigación clínica, por ser ella herramienta de formación eficaz ${ }^{13,15}$ y vía para resolver temáticas prevalentes de la realidad nacional ${ }^{18}$. Ello implica dotar de elementos que permitan adquirir habilidades en metodología científica, así como el tiempo, estímulo o exigencia para llevarla a cabo ${ }^{19}$.

La oportunidad para avanzar en esta tarea parece óptima, dado que las facultades de medicina agrupadas en ASOFAMECH han estado desde 1995 comprometidas en una revisión curricular modernizadora y en un proceso de acreditación, orientados a la satisfacción de las necesidades de la comunidad y a las demandas específicas del entorno $13,14,16,17$.

Vistas estas consideraciones, hemos querido describir las características institucionales y los procesos docentes ${ }^{17}$ en materias médico-legales que poseen las entidades formadoras nacionales. Específicamente nos interesa:

a) Obtener información metodológicamente sólida, de utilidad para la generación y revisión de programas de estudio en ML.

b) Conocer las líneas de investigación nacionales en esta materia, identificando áreas que deberán ser incorporadas y otras que deberán ser profundizadas.

c) Aportar nuestra visión como legistas de una región piloto del nuevo sistema procesal penal.

\section{MATERIAL Y MÉTODO}

Diseño. Estudio de corte transversal, realizado entre septiembre de 2003 y junio de 2004 .

Fuentes de información e instrumentos de recolección. Se abarcó el universo de facultades que imparten la carrera de medicina en el país*. Por correo electrónico, se solicitó a los decanos que completaran un cuestionario de 21 preguntas, validado previamente por contenido y fachada ${ }^{20}$, que contenía principalmente preguntas cerradas, más algunas consultas de respuesta abierta. Este cuestionario exploró diversas características de la docencia en ML en pregrado, postgrado y en otras carreras del área. En el caso de las facultades que no respondieron el cuestionario, la información se obtuvo de lo disponible en sus páginas web. Además, se les solicitó que remitieran el o los programas de ML con que cuenta cada una de las respectivas facultades. A partir de estos programas, la bibliografía ${ }^{14}$, los registros de la Corporación Nacional Autónoma de Certificación de Especialidades Médicas ${ }^{21}$ y la experiencia de los autores, se construyó una pauta estándar que permitió evaluar los procesos docentes de cada universidad.

Finalmente, mediante técnicas de bibliometría se exploraron las publicaciones chilenas sobre materias médico-legales, utilizando la base de datos especializada de la Colaboración Cochrane y las bases de datos bibliográficos universales Medline, Lilacs y SciELO Chile.

\footnotetext{
* Información oficial y vigente al 31 de diciembre de 2003. Disponible en el Directorio de Educación Superior del Ministerio de Educación, http://www.mineduc.cl.
} 
Estadística. Los datos fueron analizados con Stata 8.0. Se procedió a un análisis exploratorio descriptivo de los resultados, para lo cual se recurrió a medidas de resumen y tablas. Las comparaciones entre grupos se realizaron con la prueba exacta de Fisher. El nivel de significación utilizado en las pruebas estadísticas fue de $5 \%(\alpha=0,05)$.

El análisis de los programas de estudio se realizó mediante una pauta estándar que verificó sus contenidos con relación a 89 materias de la especialidad, agrupadas en 12 áreas temáticas. Para calificar la cantidad de contenidos de ML impartidos se utilizó una escala ordinal que permitió establecer la siguiente clasificación: "Bueno": $\geq 80 \%$ de materias, "Regular": $\geq 60 \%$ y $<80 \%$ de materias, "Insuficiente": $\geq 20 \%$ y $<60 \%$ de materias y "Malo": $<20 \%$ de materias.

Aspectos éticos. No obstante ser una investigación descriptiva, se prestó atención a requisitos éticos universalmente aplicables ${ }^{22}$. En tal sentido:

a) Se ofreció a todas las entidades formadoras la oportunidad de participar y beneficiarse de los resultados.

b) El manejo de la información fue confidencial, y no se identifica el nombre de la institución académica a la que pertenecen los resultados, evitándose con ello que sean utilizados con fines comerciales o de marketing.

c) Finalmente, para maximizar los beneficios de la investigación y obtener cambios cualitativos que lleguen concretamente a la sociedad, se envió un informe a cada facultad que participó del estudio de su situación particular dentro del contexto nacional.

\section{RESULTADOS}

A. Generalidades. La carrera de medicina se dicta en 19 universidades del país. Lo más frecuente son escuelas con más de 10 años de antigüedad (9 de 19), de carácter privado (8 de 19) y cuya casa central se ubica en regiones (10 de 19). No se observan diferencias significativas de la antigüedad de la carrera (Fischer $=0,212$ ) ni de la ubicación de su casa central (Fischer=0,111), al compararlas según el tipo de universidad (Tabla 1).

El cuestionario enviado fue respondido por 14 escuelas, que representan a $73,7 \%$ del total. De ellas, 10 enviaron sus programas de ML No hubo diferencias significativas en la colaboración al estudio al analizarla según el tipo de universidad (Fischer=0,675).

Tabla 1. Perfil de las carreras de medicina del país

\begin{tabular}{|lllc|}
\hline Nombre organismo & Tipo & Casa Central & Año creación \\
\hline Pontificia U Católica de Chile & Particular & Santiago & 1930 \\
U Austral de Chile & Particular & Valdivia & 1965 \\
UC de la Santísima Concepción & Particular & Concepción & 1997 \\
U Católica del Maule & Particular & Talca & 2003 \\
U Católica del Norte & Particular & Coquimbo & 2003 \\
U de Antofagasta & Estatal & Antofagasta & 1996 \\
U de Chile & Estatal & Santiago & 1833 \\
U de Concepción & Particular & Concepción & 1924 \\
U de La Frontera & Estatal & Temuco & 1971 \\
U de Los Andes & Privada & Santiago & 1991 \\
U de Santiago de Chile & Estatal & Santiago & 1993 \\
U de Valparaíso & Estatal & Valparaíso & 1961 \\
U del Desarrollo & Privada & Santiago & 2002 \\
U del Mar & Privada & Viña Del Mar & 2003 \\
U Diego Portales & Privada & Santiago & 2002 \\
U Finis Terrae & Privada & Santiago & 2002 \\
U Mayor & Privada & Santiago & 1998 \\
U Nacional Andrés Bello & Privada & Santiago & 2003 \\
U San Sebastián & Privada & Concepción & 1994 \\
\hline
\end{tabular}


B. Medicina legal en pregrado. La asignatura de ML está presente en forma obligatoria en todas las carreras de medicina del país. Sin embargo, en sólo 2 de ellas existe un departamento de ML. En la mayoría (13 de 19), la asignatura se dicta en forma semestral, no observándose diferencias significativas en el tiempo lectivo según tipo de universidad (Fischer $=0,876$ ) ni ubicación de la casa central (Fischer $=0,582$ ). Cabe puntualizar que una escuela posee para todas sus asignaturas la modalidad de "Aprendizaje basado en problemas" (ABP), lo que impidió en dicho caso precisar el tiempo dedicado a la docencia en ML. No fue posible establecer el número de horas o creditaje promedio de la asignatura por disparidad de criterios en las respuestas recibidas (Tabla 2).

En la mayoría de las escuelas, la asignatura se dicta durante el $5^{\mathbf{0}}$ año de la carrera (13 de 19), sólo en 2 se hace en el $4^{\circ}$ año y en otras 2 durante el internado. La modalidad de enseñanza es teóricopráctica en la mayoría de las escuelas (11 de 19), sin que existan diferencias significativas al analizarla según el tipo de universidad (Fischer=0,546).

Observamos también que en 7 de las 14 escuelas que respondieron el cuestionario se entregan conceptos de ML en otras asignaturas de pregrado, sin embargo, sólo recibimos el programa de 1 de ellas.

Basados también en las respuestas al cuestionario, verificamos que gran parte de las otras carreras de la salud no cuentan con una asignatura de ML, la cual está presente sólo en 2 de 10 carreras de odontología, 2 de 17 de enfermería, 1 de 9 de obstetricia y 1 de 9 de tecnología médica. No se dicta ML en ninguna de carrera de fonoaudiología, kinesiología y nutrición.

C. Análisis de los programas de estudio de las asignaturas de ML de pregrado del país. En las 14 escuelas de las cuales se dispuso de información, se observa que existen un total de 74 docentes de ML, 11 de los cuales realizan actividades en más de una universidad. Hay 4 universidades que aún no han contratado docentes, por cuanto todavía no dictan la asignatura; 54 docentes son médico-cirujanos $(74,0 \%)$, seguidos en frecuencia por abogados y químico-farmacéuticos (ambos 8,2\%). De los médicos docentes, 33 (61,1\%) figuran como especialistas en ML. Sin embargo, sólo $10(18,5 \%)$ están reconocidos por CONA-
CEM. Otros especialistas frecuentes son los anátomo-patólogos y psiquiatras (6 en cada especialidad) (Tabla 3).

\section{Tabla 2. Tiempo lectivo de la asignatura de medicina legal}

\begin{tabular}{|lrc|}
\hline Tiempo lectivo & № & Porcentaje $^{*}(\%)$ \\
\hline 10 días & 1 & 5,26 \\
1 mes & 1 & 5,26 \\
1 trimestre & 1 & 5,26 \\
Un semestre & 13 & 68,42 \\
Un año & 1 & 5,26 \\
ABP & 1 & 5,26 \\
Sin información & 1 & 5,26 \\
Total & 19 & 100,00 \\
\hline
\end{tabular}

${ }^{*}$ Corresponde a la frecuencia relativa de observaciones para cada categoría de tiempo lectivo, en función del total de facultades de medicina del país (19).

\section{Tabla 3. D ocentes de $M L$ en las escuelas de medicina del país}

\begin{tabular}{|lrc|}
\hline Variable & $\mathrm{n}$ & Porcentaje \\
\hline Profesión\& & & \\
Médico Cirujano & 54 & 73,97 \\
Abogado & 6 & 8,22 \\
Q. Farmacéutico & 6 & 8,22 \\
Odontólogo & 3 & 4,11 \\
Antropólogo & 2 & 2,74 \\
Bioquímico & 1 & 1,37 \\
Psicólogo & 1 & 1,37 \\
Total & 73 & 100 \\
Especialidad de los médicos & & \\
Medicina Legal & 33 & $61,11^{\#}$ \\
A. Patológica & 6 & $11,11^{\#}$ \\
Psiquiatría & 6 & $11,11^{\#}$ \\
Ginecología & 4 & $7,41^{\#}$ \\
Magíster en Ciencias & 4 & $7,41^{\#}$ \\
Otras & 17 & $31,48^{\#}$ \\
Médicos Legistas & & \\
Reconocidos por CONACEM & 10 & 30,30 \\
Sin reconocimiento & 23 & 69,70 \\
Total & 33 & 100 \\
\hline
\end{tabular}

\&Se desconoce la profesión de un docente. *Existen médicos para los cuales se registra más de una especialidad. \#Representa el porcentaje calculado en base al total de médicos docentes (54). 
El análisis de los 10 programas de estudio enviados, permitió observar que la cantidad de contenidos impartidos es insuficiente, abarcando sólo $34,4 \%$ de las 89 materias evaluadas. Además, 13,5\% de las materias no se contemplan en ninguno de los planes de estudio analizados. Las áreas temáticas mejor abordadas son "tanatología" (70,0\%), "introducción a la ML" (48,6\%) y "lesionología" (40,9\%). El tema que menos se aborda es "odontología forense" (10,0\%), seguido por "antropología forense" $(17,5 \%)$ y "psiquiatría forense" (22,5\%). El detalle de temas y materias contenidos en los programas de estudio analizados se muestra en las Tablas 4 y 5 .

D. Medicina legal en postgrado. La especialidad de ML se dicta en sólo una universidad, teniendo 2 egresados a la fecha. En ninguna de las universidades que respondieron la encuesta se dicta algún programa de postgrado (diplomado, magíster o doctorado) que tenga relación con materias médico-legales.

E. Investigación en medicina legal. Mediante bibliometría se identificaron 52 publicaciones chilenas en materias médico-legales (Tablas 6 y 7). El $59,6 \%$ de ellas está en revistas incorporadas al Index Medicus ${ }^{\circledR}$, siendo la Revista Médica de Chile la principal de ellas (46,2\%). Las áreas temáticas sobre las cuales más se publica son "Derecho Médico" (50,0\%) y "Criminalística" (19,2\%). Llama la atención que sólo en 17,3\% de las publicaciones figura como autor alguno de los docentes de ML identificados en la presente investigación.

\section{DisCUSIÓN}

Hemos observado con satisfacción que todas las carreras de medicina del país cuentan en forma obligatoria con la asignatura de ML, la que al igual que en otros países tiende a ubicarse en los últimos años de la carrera. No obstante ello, consideramos, a la luz de los resultados obtenidos, que la importancia que se le asigna a la asignatura dentro del contexto de la malla curricular de la carrera es poca. Si bien en la mayoría de las universidades la asignatura es semestral, no logramos recabar información respecto de su duración promedio, teniendo presente que lo óptimo es una duración no inferior a $65 \mathrm{~h}$ (Lencioni, Leo J, datos no publicados). Además, no en todas las universidades la

\section{Tabla 4. C antidad de materias incorporadas en planes de estudio, agrupadas por áreas temáticas}

\begin{tabular}{|lcc|}
\hline Tema & $\begin{array}{c}\text { Porcentaje } \\
(\%)\end{array}$ & Interpretación \\
\hline Introducción a la ML & 48,6 & Insuficiente \\
Introducción al derecho & 27,5 & Insuficiente \\
Tanatología & 70,0 & Regular \\
Lesionología & 40,9 & Insuficiente \\
Odontología forense & 10,0 & Malo \\
Sexología forense & 36,7 & Insuficiente \\
Toxicología forense & 31,7 & Insuficiente \\
Medicina legal laboral & 25,0 & Insuficiente \\
Psiquiatría forense & 22,5 & Insuficiente \\
Antropología forense & 17,5 & Malo \\
Criminalística & 25,0 & Insuficiente \\
Derecho médico & 34,6 & Insuficiente \\
Total & 34,4 & Insuficiente \\
\hline
\end{tabular}

modalidad de enseñanza es teórico-práctica, lo que puede atentar contra la motivación de los alumnos, quienes habitualmente se encuentran absorbidos por el estudio de materias clínicas.

El análisis de los contenidos programáticos revela que la ML que se imparte, contrario a la opinión de los otros autores sigue girando en torno a la tanatología, cuestión que a nuestro juicio se debe modificar prontamente, en el entendido que la mayoría de las acciones médicolegales que le corresponde realizar a un profesional de la salud no especialista no están en el ámbito tanatológico. A la luz de la Reforma Procesal Penal y del auge de demandas por malpraxis, creemos necesario reforzar la entrega de conocimientos en lesionología, odontología forense, criminalística y derecho médico. Quizás una forma de hacerlo sea aprovechar la modalidad de ABP, generando con ello espacios para que la entrega de contenidos en ML sea vertical a lo largo de toda la carrera.

Debe llamar la atención la escasa formación médico-legal en las demás carreras de la salud; hemos de tener presente que los problemas deontológicos afectan por igual a los distintos profesionales de la salud. Además, la actividad pericial necesita nutrirse de profesionales no médicos, los que han de poseer alguna base que los oriente y estimule a realizar formación de 
Tabla 5. G rado de abordaje de las distintas materias analizadas

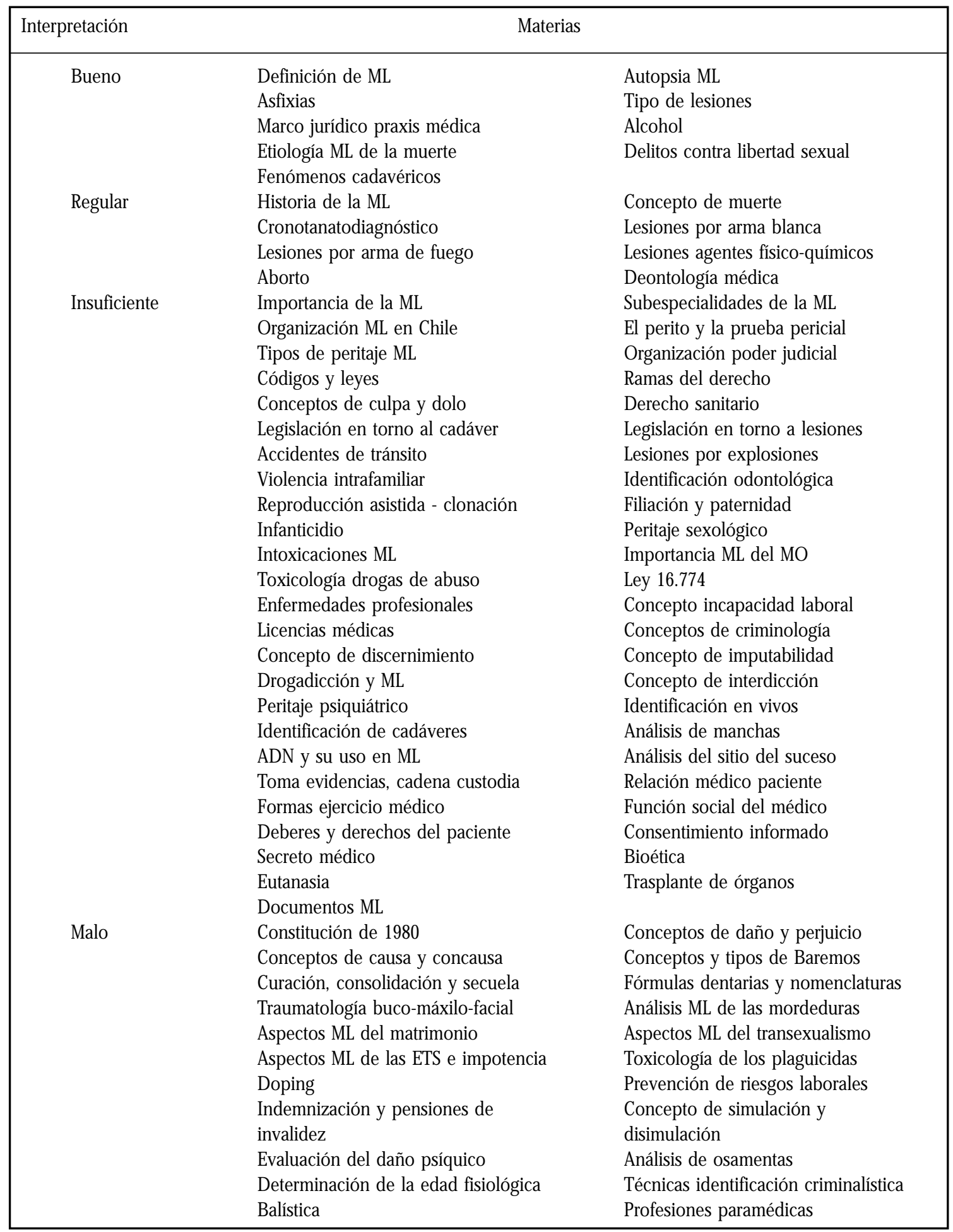


Tabla 6. Estrategia y resultados de la búsqueda sistemática de información

\begin{tabular}{|c|c|c|c|c|c|}
\hline \multirow{2}{*}{\multicolumn{2}{|c|}{ Búsqueda Estrategia\& }} & \multicolumn{4}{|c|}{ № Publicaciones encontradas } \\
\hline & & \multirow{2}{*}{$\frac{\text { Cochrane }}{0}$} & \multirow{2}{*}{$\begin{array}{r}\text { MEDLNE* }^{*} \\
53.266\end{array}$} & \multirow{2}{*}{$\begin{array}{r}\text { LILACS } \\
530\end{array}$} & \multirow{2}{*}{$\begin{array}{r}\text { SciELO } \\
6\end{array}$} \\
\hline 1 & "Forensic sciences" [MeSH] & & & & \\
\hline 2 & "Legal medicine" [tw] & 0 & 338 & 267 & 1 \\
\hline 3 & "Jurisprudence" [MeSH] & 4 & 82.459 & 3.325 & 9 \\
\hline 4 & 1 OR 2 OR 3 & 4 & 128.280 & 3.695 & 16 \\
\hline 5 & "Chile" [MeSH] OR "Chile" [tw] & 57 & 4.881 & 6.520 & 3.769 \\
\hline 6 & 4 AND 5 & 0 & 77 & 132 & 9 \\
\hline & Selección manual ${ }^{\#}$ & 0 & 23 & 22 & 7 \\
\hline
\end{tabular}

\&En Lilacs y SciELO se usaron las expresiones traducidas a partir del índice de palabras. *Se limitó a publicaciones en humanos. "Realizada en base a títulos, abstract y excluyendo referencias repetidas.

postgrado en el área. Ello implica también la necesidad de expandir la precaria formación de postgrado existente en la actualidad, creando nuevos centros formadores de especialistas en ML, incorporando créditos de ML en todos los programas de formación de especialistas y desarmollando programas de post-título y de formación continua abiertos a la participación multi e interdisciplinaria. A nuestro entender dichas acciones requieren, entre otros, que el Ministerio de Justicia asuma un activo rol en su financiamiento.

Quizás uno de los principales desafíos por abordar es la investigación en ML. La cantidad de publicaciones existentes es absolutamente deficiente y en su mayonía no se generan desde centros docentes. Además, buena parte de lo publicado son revisiones no sistemáticas ("Review"), estudios de laboratorio, serie de casos o cartas al editor. Prueba de ello es que entre 1995 y 2000 no existió ningún proyecto FONDECYT en el área de $\mathrm{ML}^{18}$. Esto significa que el nivel de evidencia aportado por las publicaciones nacionales tiende a ser bajo, cuestión que habrá que mejorar a la luz de las experticias y conocimientos necesarios para enfrentar el nuevo sistema procesal penal. En tal sentido, surge como necesidad la creación de centros de investigación en ML y la incorporación de epidemiólogos clínicos e investigadores del área de las ciencias sociales a los equipos docentes actualmente existentes en el país, por cuanto no podemos depender de resultados de investigaciones foráneas que no siempre son aplicables a la realidad nacional ${ }^{19}$.

$\mathrm{Si}$ bien reconocemos que nuestra investigación posee sesgo de voluntario por el hecho de no haber recibido respuesta al cuestionario por parte
Tabla 7. Publicaciones en medicina legal realizadas por profesionales chilenos

\begin{tabular}{|lrc|}
\hline Variable & № & Porcentaje (\%) \\
\hline Área temática & & \\
Derecho médico & 26 & 50,00 \\
Criminalística & 10 & 19,23 \\
Psiquiatría forense & 7 & 13,46 \\
Sexología forense & 3 & 5,77 \\
Tanatología & 3 & 5,77 \\
Odontología forense & 2 & 3,85 \\
Lesionología & 1 & 1,92 \\
Total & 52 & 100 \\
Lugar de publicación & & \\
Rev Méd Chile & 24 & 46,15 \\
J Forensic Sci & 6 & 11,54 \\
Rev Chil Psiquiatría & 6 & 11,54 \\
Otras & 16 & 30,77 \\
Total & 52 & 100 \\
Docente de ML & & \\
Si & 9 & 17,31 \\
No & 43 & 82,69 \\
Total & 52 & 100 \\
\hline
\end{tabular}

de 5 universidades, consideramos que sus resultados son válidos, al haber abarcado casi $75 \%$ del universo de carreras de medicina del país. Esperamos por tanto que los resultados aquí presentados resulten útiles para generar las bases que permitan unificar los programas y modalidades de enseñanza, atendiendo a los desafíos que hemos comentado. Bien lo dice Jouvencel: "la atención que un país presta a la medicina forense es un exponente de madurez social, un índice de civismo"23. 


\section{REFERENCIAS}

1. Rojas, Nerio. Medicina Legal. 12 ${ }^{\mathrm{a}}$ ed. El Ateneo. Buenos Aires. 1984.

2. Sateler R, Carrasco S, Castro JL, Nancuante U. Exposiciones en Seminario "La Práctica Médica en Chile y la Judicialización de la Medicina en el contexto de la Reforma de Salud", Santiago, Abril 2003. Disponibles en http:// www.saludyfuturo.cl

3. Montoya D, Rosmanich A, Velásquez V, López J. Querellas por responsabilidad médica según especialidades en Chile. Rev Méd Chile 1993; 121: 396-402.

4. Montoya D, Rosmanich A, Velásquez V, López J. Aspectos médicos y legales de las querellas por responsabilidad médica. Rev Méd Chile 1993; 121: 679-85.

5. Montoya D, Rosmanich V, López J. Aspectos clínicos y evaluación médico legal en demandas por responsabilidad médica en obstetricia y ginecología. Rev Chil Obstet Ginecol 1990; 55: 403-12.

6. Court E. Algunas consideraciones sobre la responsabilidad civil médica a la luz de la doctrina y jurisprudencias nacionales. Bol Hosp Viña del Mar 1996; 52 (4).

7. Teke A, Navarro R, Cerda C, Lam M, Lagos E, GuZMán M ET AL. Demandas judiciales contra médicos en Chile. Rev Méd Chile 1996; 124: 33747.

8. Von Bennewitz R. Las demandas y querellas por responsabilidad médica en la hora actual y sus mecanismos de regulación. Cuad Méd Soc, XXXIV, 2 y 3, 1993/15-28.

9. La Judicialización de la Medicina en Chile. Revista Vida Médica, Volumen 55, № 1, 2003.

10. Ley Orgánica del Servicio Médico Legal. Artículo 22. Disponible en: http://www.sml.cl/normativas. $\mathrm{htm} \#$

11. Nuevo Código Procesal Penal. Artículos 198, 199, 200. Edición oficial. Editorial Jurídica de Chile.

12. Ley 18.355. Diario Oficial, 07 de noviembre de 1984.

13. PINTO ME. Formación de médicos especialistas: un desafío permanente. Publicación on line del Consejo Superior de Educación. En: http:// www.cse.cl/Publicaciones/Calidad/0103/PDF/ Pinto.pdf
14. RosseLot JE. Hacia el médico que nuestros países necesitan: énfasis en la comunicación en la formación de los docentes. Rev Méd Chile 2003; 131: 331-7.

15. Román AO. Un análisis de la situación actual de las especialidades médicas en Chile. Rev Méd Chile 2002; 130: 809-15.

16. Rosselot JE. Planificando la educación médica para las próximas décadas. Rev Méd Chile 2001; 129: 1473-8.

17. Rosseiot JE. Aseguramiento de la calidad profesional. Un nuevo marco ético para el ejercicio de la medicina. Rev Méd Chile 1999; 127: 1375-83.

18. NoRERo VC, RosSeLot JE. Investigación clínica en Chile: ¿Cómo superar una inquietante evolución? Rev Méd Chile 2001; 129: 317-23.

19. Stockins FB. La Investigación en Salud en Chile. Rev Méd Chile 2000; 128: 1389-95.

20. Streiner DL, Norman GR. Health measurement scales: a practical guide to their development and use. Oxford: Oxford University Press, 1989.

21. CONACEM. Registro de Especialistas en Medicina Legal. En: http://www.conacem.cl

22. EManuel E. ¿Qué hace que la investigación sea ética? Siete requisitos básicos. En Pellegrini A, Macklin R. (eds.), Investigaciones en sujetos humanos: experiencia internacional. Serie de Publicaciones Programa regional de bioética OPS/OMS, Santiago de Chile, Impresos Universitaria SA, 1997; 33-46.

23. Jouvencel MR. Manual del Perito Médico. Ed. Díaz de Santos S.A., Madrid. 2002.

Agradecimientos:

Queremos expresar nuestros más sinceros agradecimientos a las 14 escuelas de medicina del país que participaron de esta investigación. Particularmente nos interesa agradecer al Dr. Wilfred Diener O. quien consiguió que esta investigación fuera patrocinada por ASOFAMECH. También a los Dres. Marcos Cikutovic S., Luis Ciocca G., Héctor Figueroa M., Rodrigo Valdés A., Nicolás Velasco M. y Santiago Soto O., por sus elogiosos comentarios, colaboración y muestras de apoyo. Especial mención nos merece también el Dr. Leo Julio Lencioni, quien tuvo la amabilidad de remitirnos los resultados íntegros de la investigación que presentó en Buenos Aires durante el Congreso "Forense Argentina 2003", y que constituyó un importante referente bibliográfico. 